\title{
Multi-boson Production in Weak Boson Fusion
}

\author{
Michael Rauch* \\ Institute for Theoretical Physics, Karlsruhe Institute of Technology (KIT), Germany \\ E-mail: michael.rauch@kit.edu
}

\section{Francisco Campanario}

Theory Division, IFIC, University of Valencia-CSIC, E-46980 Paterna, Valencia, Spain

E-mail: francisco.campanariodific.uv.es

\section{Bastian Feigl}

Institute for Theoretical Physics, Karlsruhe Institute of Technology (KIT), Germany

\section{Oliver Schlimpert}

Institute for Theoretical Physics, Karlsruhe Institute of Technology (KIT), Germany

\begin{abstract}
The production of multiple gauge bosons via weak boson fusion is an important process at the LHC. It is relevant as a background process appearing in many searches and measurements, but also serves as a signal process when studying new-physics contributions to triple and in particular quartic gauge boson vertices.

We first review the theoretical status of multi-boson production in weak boson fusion and present the current state of the art. In the second part, the impact of anomalous gauge couplings on this class of processes is discussed.
\end{abstract}

XXII. International Workshop on Deep-Inelastic Scattering and Related Subjects, 28 April - 2 May 2014

Warsaw, Poland

\footnotetext{
*Speaker.
} 

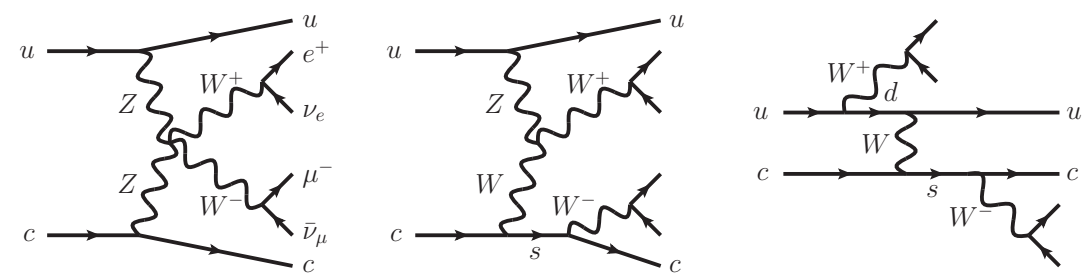

Figure 1: Example Feynman diagrams for $W^{+} W^{-}$production via VBF including leptonic decays of the gauge bosons.

\section{Introduction}

The production of multiple bosons in weak-boson fusion, often also called vector-boson fusion (VBF) or vector-boson scattering, yields a distinct signature, which is characteristic for the whole class of weak-boson fusion processes. It consists of two jets in the forward region of the detector, the so-called tagging jets, and a reduced jet activity in the central region. The leptonic decay products of the final-state bosons are typically in rapidity between the tagging jets. This process class can also be seen as a two-sided deep-inelastic scattering (DIS) process, where the electron side of the DIS process is replaced by the other quark in each case.

The process class of weak-boson fusion has first been studied in the context of Higgs searches [1], where the two tagging jets serve as an experimental trigger and allow for a reduction of background processes. Later on, the focus has been extended to VBF production of single gauge bosons $(W$, $Z, \gamma)[2]$ and finally to diboson production [3-10]. In this overview, we will restrict ourselves to the production of a pair of gauge bosons, since the Higgs-pair production process is discussed in a separate article [11]. These studies include the leptonic decays of the gauge bosons as well as off-shell and non-resonant contributions.

A set of typical Feynman diagrams is shown in Fig. 1, taking $W^{+} W^{-}$production via VBF as an example process. The final-state $W$ can either be attached directly to the quark lines, as in the diagram on the right-hand side, or one or both are attached to the intermediate t-channel gauge boson. This gives then rise to triple and quartic gauge boson vertices, as shown in the middle or left-hand Feynman diagrams, respectively. Therefore, the non-Abelian structure of the weak interaction can be studied with these processes. Additionally, they give access to triple and, in particular, quartic anomalous gauge couplings [12, 13].

Furthermore, these processes form a background for many collider searches, both within the Standard Model and beyond. For example, they constitute an irreducible background to VBF production of Higgs bosons where the Higgs decays into gauge bosons. They are important both for the discovered light Higgs state with a mass of around $125 \mathrm{GeV}$, but also for searches for heavy Higgs bosons. There, a possibly large width of the heavy Higgs can lead to significant interference effects between the Higgs signal and the continuum background diagrams [14].

On the experimental side, evidence for VBF production of the single gauge boson process $Z j j$ [15] and the diboson process $W^{ \pm} W^{ \pm} j j[16]$ has been established so far by the ATLAS experiment. The latter process has also been studied by CMS [17], where the observed significance does not yet reach the $3 \sigma$ level. 


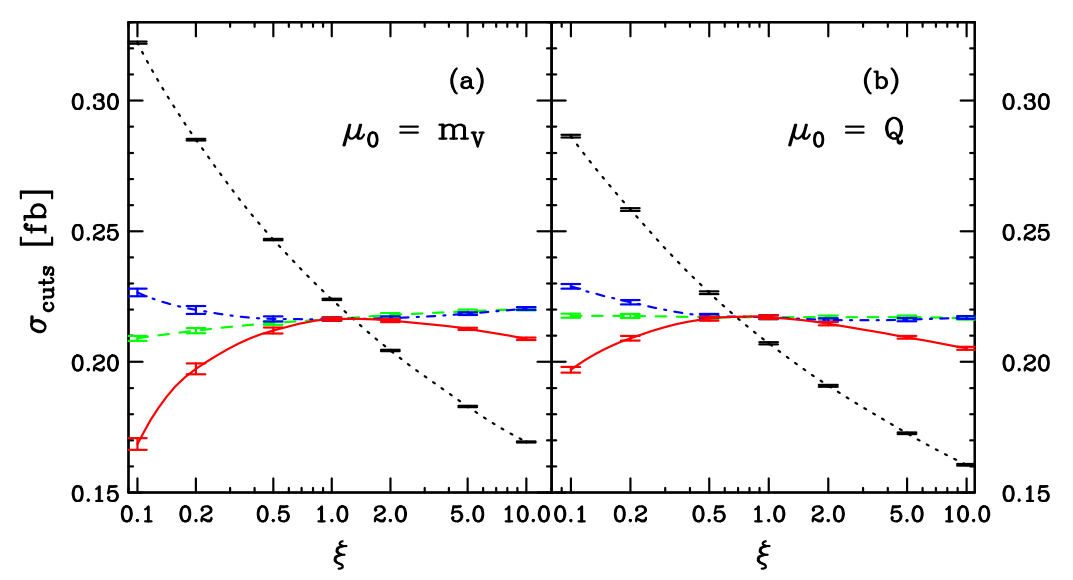

Figure 2: Dependence of the VBF- $W^{+} Z j j$ production cross sections on the scale choice $\mu=\xi \mu_{0}$. Results are shown for LO (dotted black), which only depends on the factorization scale, and for NLO when varying the factorization scale only (dot-dashed blue), the renormalization scale only (dashed green), or both jointly (solid red). Left: Fixed scale $\mu_{0}=m_{V}=\frac{m_{W}+m_{Z}}{2}$, right: momentum transfer $\mu_{0}=Q$. Figure from Ref. [5].

\section{Higher-order corrections and parton-shower effects}

Next-to-leading-order (NLO) QCD corrections for diboson production via VBF have been calculated in Refs. $[3-6,8,10]$. Most of these calculations have been performed in the so-called VBF approximation. There, contributions from s-channel diagrams, which can also be viewed as triboson production processes where one of the gauge bosons decays hadronically, as well as effects from interference between $t$ - and $u$-channel diagrams are neglected. This approximation has been checked explicitly at leading order (LO) by performing the full calculation of electroweak $W^{+} W^{+} j j$ production in Ref. [8], and extending this comparison also to the QCD-induced production process in Ref. [18]. When applying VBF cuts, which exploit the typical structure of these processes, $m_{j_{1} j_{2}}>600 \mathrm{GeV}, y_{j_{1}} \times y_{j_{2}}<0,\left|y_{j_{1}}-y_{j_{2}}\right|>4$ and $y_{j_{\min }}<y_{\ell}<y_{j_{\max }}$, where $j_{1}$ and $j_{2}$ denote the first and second hardest jet, the difference between the full calculation and the VBF-approximated one becomes negligible. This set of cuts also allows for an efficient suppression of the corresponding QCD-induced processes, which have been studied in much detail in Refs. $[18,19]$.

The dependence on the renormalization and factorization scales at LO and NLO is shown exemplarily for $W^{+} Z j j$ VBF-production in Fig. 2. The LO cross section shows a sizable scale dependence of $\pm 10 \%$ when varying the scale between $\frac{1}{2}$ and 2 times the central value. At NLO this is strongly reduced to a value of $\pm 2 \%$ for integrated cross sections and up to $\pm 6 \%$ in distributions. Thereby, taking the dynamic scale $Q$, defined as the momentum transfer of the exchanged vector boson in VBF diagrams, for each quark line, proves advantageous. As one can see in the figure, both at LO and NLO the scale variation is reduced compared to a fixed scale. The $K$ factor is close to unity for both scale choices. In differential distributions, the NLO QCD corrections can lead to relevant changes in shape. Therefore, a simple rescaling with the integrated $\mathrm{K}$ factor is not sufficient. Also here, choosing the momentum transfer as a scale leads to flatter differential $\mathrm{K}$ factors than a fixed scale. A dedicated Monte Carlo tool to calculate these processes at NLO QCD 

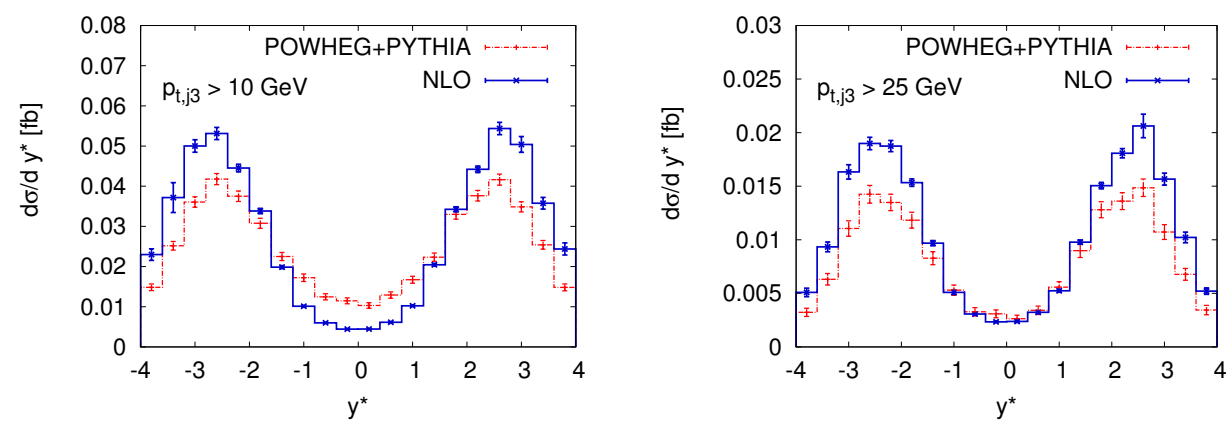

Figure 3: Differential cross section of the process $W^{+} W^{-} j j$ for the relative position of the third-hardest jet with respect to the two tagging jets, $y^{*}$, for the pure NLO QCD calculation (solid blue) and interfaced with a parton shower (dot-dashed red). Shown are results for two different values of the minimum transverse momentum of the third jet. Figure taken from Ref. [22].

at a fully differential level is available with the program VBFNLO [20].

The next step is then to interface these NLO calculations with a parton shower, which combines the advantages of both approaches. The normalization of the cross section is correct to NLO and the emission of the extra, third, jet is accurately described also when it possesses large transverse momentum. At the same time, the Sudakov suppression at small transverse momentum is correctly modeled by the parton shower, and it is possible to generate events not only at the parton, but also at the hadron level. Results have been presented for $W^{+} W^{+} j j$ [21], $W^{+} W^{-} j j$ [22] and $Z Z j j$ [23] production via VBF. All calculations available so far make use of the POWHEG-BOX tool [24], which employs the POWHEG formalism [25] to combine NLO calculations and parton shower.

In Fig. 3 we show effects of the parton shower on the emission of the extra jet for $W^{+} W^{-} j j$ production via VBF. The kinematic variable studied is the relative position of the third-hardest jet with respect to the two tagging jets, $y^{*}=y_{j_{3}}-\frac{y_{j_{1}}+y_{j_{2}}}{2}$. Imposing only a loose cut on its transverse momentum, $p_{T, j_{3}}>10 \mathrm{GeV}$, displayed on the left-hand side, we see that compared to the pure NLO calculation, the parton shower predicts extra jet activity in the central rapidity region between the two tagging jets. In contrast, the cross section for emission close to one of the tagging jets is reduced. The extra emission in the central region has mostly small transverse momentum, as can be seen from the comparison with the plot on the right-hand side, where the minimum $p_{T, j_{3}}$ has been increased to $25 \mathrm{GeV}$. There, the predictions with and without parton shower agree in the central region. An important technique in separating VBF processes from the corresponding QCD-induced ones is applying a so-called mini-jet veto, i.e. requiring that no extra emission above a certain, typically rather small, minimal transverse momentum takes place in the region between the two tagging jets. These results show that parton-shower effects can induce relevant differences compared to a pure NLO QCD calculation.

\section{Anomalous gauge couplings}

The appearance of quartic gauge boson vertices makes diboson production via weak-boson fusion an excellent process to test anomalous contributions to these vertices. A helpful tool to describe the deviations from the Standard Model (SM) prediction in a consistent, Lorentz- and gauge-symmetry conserving way is the use of effective field theory (EFT). There, new-physics 

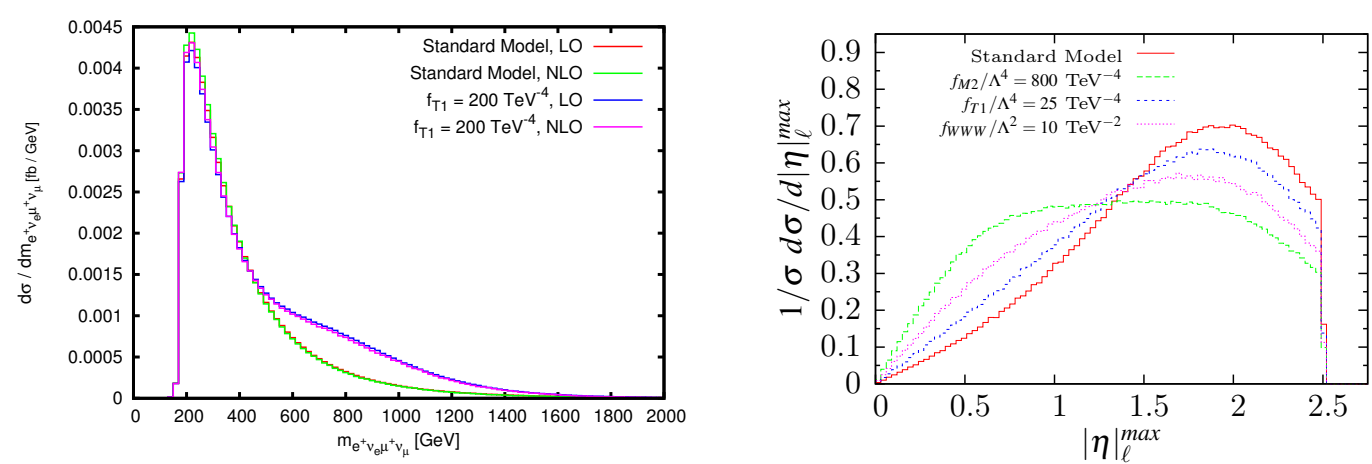

Figure 4: Left: Differential cross section for the invariant mass distribution of the two lepton, two neutrino system in VBF- $W^{+} W^{+} j j$ production with and without anomalous $T_{1}$ coupling at LO and NLO. Figure taken from Ref. [13]. Right: Normalized differential cross section for the pseudorapidity of the leading lepton in VBF- $W^{+} W^{-} j j$ production for the SM and three different operators of anomalous gauge couplings. Figure taken from Ref. [28].

contributions are assumed to be too heavy to produce them directly, but their effects can modify the interactions between the SM particles and appear as higher-dimensional operators in the Lagrangian: $\mathscr{L}_{E F T}=\mathscr{L}_{S M}+\sum_{d>4} \sum_{i} \frac{c_{i}}{\Lambda^{d-4}} \mathscr{O}_{i}$, where $d$ is the dimension of the operators and $\Lambda$ the scale of new physics. The building blocks for the operators $\mathscr{O}_{i}$, which are Lorentz scalars and gauge singlets, are the field strength tensors of the gauge fields, the Higgs and fermion fields, and partial and covariant derivatives. Below $\Lambda$, where this approach is valid, only the operators with lowest dimensions can give large contributions and should therefore be kept. For the VBF processes, these are the operators of dimension 6 and dimension $8[12,13]$. The former can also be constrained by processes with triple gauge boson vertices only, while the bosonic operators of the latter generate only vertices with at least four bosons. Therefore they are particularly relevant for VBF.

If relevant scales of the process become larger than $\Lambda$, the EFT approach breaks down. An indication for this can be the violation of unitarity in the scattering amplitude. In experimental searches one has to make sure that the sensitivity is not driven by phase-space regions where unitarity is violated. Several possibilities for this task are possible, one of the simplest being a step function which turns off anomalous couplings for large energies. Other approaches just dampen the additional contributions and give a smoother cut-off. Examples are the K-matrix method [26], where the amplitude is projected such that its value is exactly at the unitarity bound, or a dipole form factor $\left(1+\frac{m_{V V}}{\Lambda_{F F}^{2}}\right)^{-p}$, where the exponent $p$ is an additional free variable. The last approach is used for the results in the following. A dedicated tool to calculate the bound where unitarity violation happens and a corresponding dipole form factor is available at Ref. [27].

On the left-hand side of Fig. 4 we first show the influence of anomalous couplings on the NLO QCD corrections for $W^{+} W^{+}$production via VBF. As operator we choose $\mathscr{O}_{T_{1}}=\left[W^{\alpha v} W_{\mu \beta}\right] \times$ $\left[W^{\mu \beta} W_{\alpha \nu}\right]$ as an example. Starting at an invariant $W^{+} W^{+}$mass of about $500 \mathrm{GeV}$, the additional contributions due to the anomalous coupling lead to an increase of the cross section, before the dipole form factor with $\Lambda_{F F}=1188 \mathrm{GeV}$ and $p=4$ damps the contribution again at higher invariant masses. The effect of NLO corrections is small both without and with anomalous couplings and does not influence the extraction of anomalous couplings. The right-hand side of Fig. 4 presents the 
normalized differential cross section for the pseudorapidity of the leading lepton in VBF- $W^{+} W^{-} j j$ production. Besides the SM, results are also shown for the dimension-8 operators $\mathscr{O}_{T_{1}}$ and $\mathscr{O}_{M_{2}}=$ $\left[B^{\mu v} B_{\mu v}\right] \times\left[\left(D^{\beta} \Phi\right)^{\dagger}\left(D_{\beta} \Phi\right)\right]$ as well as the dimension-6 operator $\mathscr{O}_{W W W}=\operatorname{Tr}\left[W_{\mu v} W^{v \rho} W_{\rho}{ }^{\mu}\right]$. The anomalous couplings always lead to a shift from larger to smaller pseudorapidities, but the size of the shift and therefore the shape of the distributions differs for the various operators. This feature could eventually be used to distinguish different operators once a deviation from the SM prediction has been established.

\section{Conclusions}

Diboson production via weak-boson fusion is an important process for the LHC. It is a background to many searches and measurements, both within and beyond the Standard Model. Due to the appearance of Feynman graphs with quartic gauge boson vertices it can also be used as a signal process in its own right and allows to study anomalous triple and in particular quartic gauge couplings. NLO QCD corrections have been calculated and turn out to be modest. Choosing the momentum transfer via the t-channel gauge bosons as a scale thereby proves advantageous. For a number of processes also a combination with a parton shower is available. These show enhanced rates in the central region for the third-hardest jet at low transverse momentum. Experimental results for the VBF processes have started to appear only very recently due to still rather small numbers of events collected so far at the LHC. The prospects to study these processes in more detail at the upcoming run of the LHC and other future hadron colliders are very promising.

\section{Acknowledgments}

We would like to thank the organizers for the invitation and the stimulating atmosphere at the conference. We would also like to thank D. Zeppenfeld for many helpful discussions. MR acknowledges support by the Deutsche Forschungsgemeinschaft via the Sonderforschungsbereich/Transregio SFB/TR-9 “Computational Particle Physics".

\section{References}

[1] V. D. Barger, R. J. N. Phillips and D. Zeppenfeld, Phys. Lett. B 346 (1995) 106; V. Del Duca, G. Klamke, D. Zeppenfeld, M. L. Mangano, M. Moretti, F. Piccinini, R. Pittau and A. D. Polosa, JHEP 0610 (2006) 016; T. Han, G. Valencia and S. Willenbrock, Phys. Rev. Lett. 69 (1992) 3274; T. Figy, C. Oleari and D. Zeppenfeld, Phys. Rev. D 68 (2003) 073005; M. Ciccolini, A. Denner and S. Dittmaier, Phys. Rev. Lett. 99 (2007) 161803; M. Ciccolini, A. Denner and S. Dittmaier, Phys. Rev. D 77 (2008) 013002; P. Bolzoni, F. Maltoni, S. -O. Moch and M. Zaro, Phys. Rev. Lett. 105 (2010) 011801; T. Figy, S. Palmer and G. Weiglein, JHEP 1202 (2012) 105; P. Bolzoni, F. Maltoni,

S. -O. Moch and M. Zaro, Phys. Rev. D 85 (2012) 035002;

[2] D. L. Rainwater, R. Szalapski and D. Zeppenfeld, Phys. Rev. D 54 (1996) 6680; C. Oleari and D. Zeppenfeld, Phys. Rev. D 69 (2004) 093004; B. Jäger, Phys. Rev. D 81 (2010) 114016.

[3] B. Jäger, C. Oleari and D. Zeppenfeld, JHEP 0607 (2006) 015.

[4] B. Jäger, C. Oleari and D. Zeppenfeld, Phys. Rev. D 73 (2006) 113006. 
[5] G. Bozzi, B. Jäger, C. Oleari and D. Zeppenfeld, Phys. Rev. D 75 (2007) 073004.

[6] B. Jäger, C. Oleari and D. Zeppenfeld, Phys. Rev. D 80 (2009) 034022.

[7] K. Arnold, T. Figy, B. Jäger and D. Zeppenfeld, JHEP 1008 (2010) 088.

[8] A. Denner, L. Hosekova and S. Kallweit, Phys. Rev. D 86 (2012) 114014.

[9] J. Baglio, A. Djouadi, R. Gröber, M. Mühlleitner, J. Quevillon and M. Spira, JHEP 1304 (2013) 151.

[10] F. Campanario, N. Kaiser and D. Zeppenfeld, Phys. Rev. D 89 (2014) 014009.

[11] J. Baglio, PoS (DIS2014) 120.

[12] W. Buchmuller and D. Wyler, Nucl. Phys. B 268 (1986) 621; K. Hagiwara, R. D. Peccei, D. Zeppenfeld and K. Hikasa, Nucl. Phys. B 282 (1987) 253; K. Hagiwara, S. Ishihara, R. Szalapski and D. Zeppenfeld, Phys. Lett. B 283 (1992) 353; K. Hagiwara, S. Ishihara, R. Szalapski and D. Zeppenfeld, Phys. Rev. D 48 (1993) 2182; C. Arzt, M. B. Einhorn and J. Wudka, Nucl. Phys. B 433 (1995) 41; J. Wudka, Int. J. Mod. Phys. A 9 (1994) 2301; O. J. P. Eboli, M. C. Gonzalez-Garcia and J. K. Mizukoshi, Phys. Rev. D 74 (2006) 073005; B. Grzadkowski, M. Iskrzynski, M. Misiak and J. Rosiek, JHEP 1010 (2010) 085; C. Degrande, N. Greiner, W. Kilian, O. Mattelaer, H. Mebane, T. Stelzer, S. Willenbrock and C. Zhang, Annals Phys. 335 (2013) 21.

[13] C. Degrande, O. Eboli, B. Feigl, B. Jäger, W. Kilian, O. Mattelaer, M. Rauch et al., arXiv:1309.7890 [hep-ph].

[14] LHC Higgs Cross Section Working Group Collaboration, arXiv:1307.1347 [hep-ph].

[15] G. Aad et al. [ATLAS Collaboration], JHEP 1404 (2014) 031.

[16] G. Aad et al. [ ATLAS Collaboration], arXiv:1405.6241 [hep-ex].

[17] CMS Collaboration [CMS Collaboration], CMS-PAS-SMP-13-015.

[18] F. Campanario, M. Kerner, L. D. Ninh and D. Zeppenfeld, Phys. Rev. D 89 (2014) 054009.

[19] T. Melia, K. Melnikov, R. Rontsch and G. Zanderighi, JHEP 1012 (2010) 053; T. Melia, K. Melnikov, R. Rontsch and G. Zanderighi, Phys. Rev. D 83 (2011) 114043; N. Greiner, G. Heinrich, P. Mastrolia, G. Ossola, T. Reiter and F. Tramontano, Phys. Lett. B 713 (2012) 277; F. Campanario, M. Kerner, L. D. Ninh and D. Zeppenfeld, Phys. Rev. Lett. 111 (2013) 5, 052003 and arXiv:1402.0505 [hep-ph] and arXiv:1405.3972 [hep-ph]; T. Gehrmann, N. Greiner and G. Heinrich, Phys. Rev. Lett. 111 (2013) 22, 222002; Z. Bern, L. J. Dixon, F. Febres Cordero, S. Hoeche, H. Ita, D. A. Kosower, N. A. Lo Presti and D. Maitre, arXiv:1402.4127 [hep-ph]; J. Alwall, R. Frederix, S. Frixione, V. Hirschi, F. Maltoni, O. Mattelaer, H. -S. Shao and T. Stelzer et al., arXiv:1405.0301 [hep-ph].

[20] D. Zeppenfeld et al., Comput. Phys. Commun. 180 (2009) 1661; arXiv:1107.4038 [hep-ph]; arXiv:1207.4975 [hep-ph]; arXiv:1404.3940 [hep-ph].

[21] B. Jäger and G. Zanderighi, JHEP 1111 (2011) 055.

[22] B. Jäger and G. Zanderighi, JHEP 1304 (2013) 024.

[23] B. Jäger, A. Karlberg and G. Zanderighi, JHEP 1403 (2014) 141.

[24] S. Alioli, P. Nason, C. Oleari and E. Re, JHEP 1006 (2010) 043.

[25] P. Nason, JHEP 0411 (2004) 040; S. Frixione, P. Nason and C. Oleari, JHEP 0711 (2007) 070.

[26] A. Alboteanu, W. Kilian and J. Reuter, JHEP 0811 (2008) 010.

[27] http://www.itp.kit.edu/ vbfnloweb/wiki/doku.php?id=download:formfactor

[28] O. Schlimpert, diploma thesis, 2013. http://www.itp.kit.edu/prep/diploma/PSFiles/Diplom_Schlimpert.pdf 